\title{
Comparing Original and Encrypted Sizes of Colorful Images by Steganography
}

\author{
C. Tiken, R. Samli, and S. Sevgen
}

\begin{abstract}
In today's digital world the secure and confidential data transmission by the various ways is quite vital. In order to avoid attacks which aim to receive data unauthorizedly, many different techniques have been developed by the years. In this paper one of these techniques called "image steganography" is analyzed. Ten colourful basic and popular images of image processing were used for the experiments. Also an image to hide in these ten images is chosen. The filtering algorithms is "mean filter algorithm". This filtered hidden image also has been hidden in these ten images. After that, the physical sizes of images were compared after encryption process. MATLAB program has been used for all these issues.
\end{abstract}

Keywords-Image Processing, Mean Filter, Image Steganography.

\section{INTRODUCTION}

In computer area, image processing uses digital images. A digital image is made up of arrangement of numbers that represent light intensities at a range of points called "pixels". A digital image is collection of pixels which can be thought of small dots on the screen. Pixels give the properties of images [1].

As it is known that network attacks are increasing day by day by using new techniques. To avoid attacks from any unauthorized people/company/system and so on, many different contra techniques and approaches have been developed to make data over a network confidential and durable [2]. For transmission of secret information from one place to another place for different application steganography is one of the most commonly used counter technique.

A type of steganography "image steganography" is a popular steganography type for hiding data since it provides a secure and simple way to send the information over the internet [3].

\section{SteganograPHy AND MEAN FILTER}

Steganography is the art of hiding the information which is to be communicated in other information. Most commonly used file format for communication is digital image due its frequency on the Internet. For hiding secret information in images, there

Manuscript received Nov. 2, 2019. This work was supported in part by the İstanbul Arel University.

C. Tiken is with the İstanbul Arel University, Department of Computer Engineering, İstanbul, TK 34537 Turkey.

R. Samli is with Istanbul University-Cerrahpasa,, Department of Computer Engineering, İstanbul, TK 34320 Turkey..

S. Sevgen is with Istanbul University-Cerrahpasa,, Department of Computer Engineering, İstanbul, TK 34320 Turkey. exists a large variety of steganographic techniques some are more complex than others and all of them have respective strong and weak points.

Different applications have different requirements of the steganography technique used. For example, some applications may require absolute invisibility of the secret information, while others require a larger secret message to be hidden. Steganography can be classified into four types: text, image, audio and video. In this paper it is shown how an image can be hidden in another image.

Images are routinely used in diverse areas such as medical, military, science, engineering, advertising, education as well as training. With the increasing use of digital techniques for transmitting and storing images, the fundamental issue of protecting the confidentiality, Integrity as well as the authenticity of images has become a major concern [2].

Mean filter is a simple sliding-window spatial filter that replaces the center value in the window with the average (mean) of all the pixel values in the window. The window, or kernel, is usually square but can be any shape. An example of mean filtering of a single $3 \times 3$ window of values is shown below in Fig. $1[4][5][6][7]$.

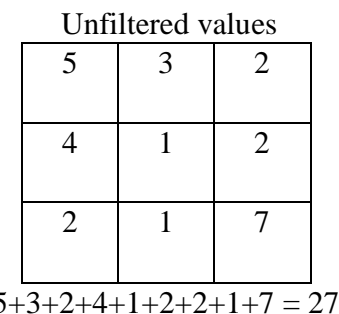

$27 / 9=3$

Mean filtered

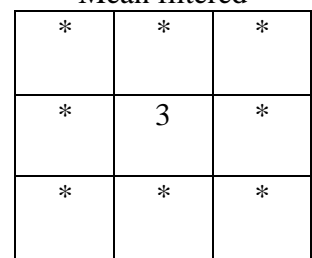

Fig. 1. Center value (previously 1) is replaced by the mean of all nine values (3).

\section{APPLICATION}

In this paper, some popular images in image processing researches were used. First the hidden image which is cube.png and original size is $142 \times 131$ pixels Fig.2. was determined. Cover images are shown in Fig. 3. each of them is colorful and has 512x512 pixels as size also in the Portable Network Graphic (PNG) format. 


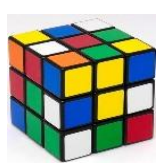

Fig. 2. Colorful hidden 142x131 pixels cube.png image.

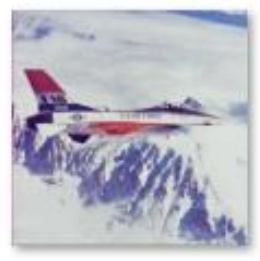

Airplane

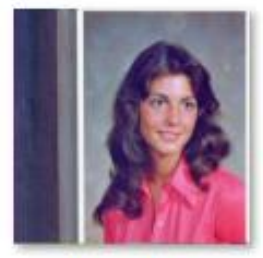

Female

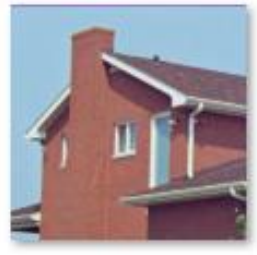

House

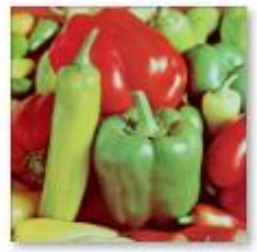

Peppers

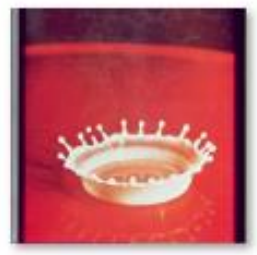

Splash

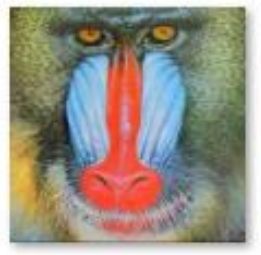

baboon

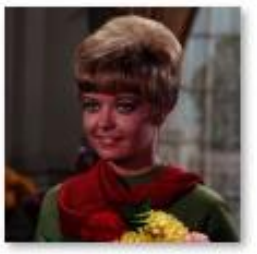

Female_2

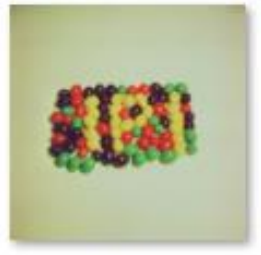

Jelly beans

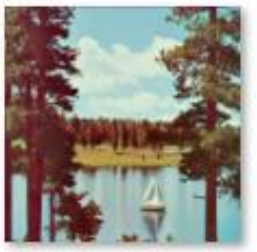

Sailboat

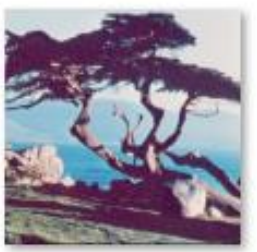

Tree
Fig. 3. Ten most popular colorful 512x512 pixels "png" format cover images.

The physical size (kilobyte) of original images, encrypted image which hides the original hidden image and encrypted image which hides mean filtered hidden image were compared. Mean filtering was applied to each $8 \times 8$ pixel blocks of the original hidden image so that we obtained a complete mean filtered hidden image Fig. 4.

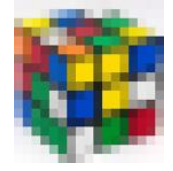

Fig. 4. mean filtered hidden image

\section{EXPERIMENTS AND RESULTS}

The experiments in this paper were done using MATLAB to explore the efficiency of this experiment. Encryption process applied to each images respectively and their physical sizes before any steganographic encryption and after two different encryption observed. Also the sizes of original images were given. The results show that the mean filtering does not make any sense at seven images. Size of the three images with original hidden image have more size. And interestingly the encrypted baboon.png image's size is smaller than the original size. Results were shown in Table 1.

TABLE 1. COMPARISON OF ENCRYPTED IMAGES (STEGO-IMAGE) WITH HIDDEN AND ENCRYPTED IMAGES (STEGO-IMAGE) WITH FILTERED HIDDEN

\begin{tabular}{llll}
\hline Image & Physical size & $\begin{array}{l}\text { Stego-image } \\
\text { with original } \\
\text { hidden }\end{array}$ & $\begin{array}{l}\text { Stego-image } \\
\text { with filtered } \\
\text { hidden }\end{array}$ \\
\hline Tree.png & $379 \mathrm{~KB}$ & $391 \mathrm{~KB}$ & $392 \mathrm{~KB}$ \\
Splash.png & $384 \mathrm{~KB}$ & $388 \mathrm{~KB}$ & $388 \mathrm{~KB}$ \\
Sailboat.png & $535 \mathrm{~KB}$ & $536 \mathrm{~KB}$ & $536 \mathrm{~KB}$ \\
Peppers.png & $495 \mathrm{~KB}$ & $496 \mathrm{~KB}$ & $496 \mathrm{~KB}$ \\
Jellybeans.png & $166 \mathrm{~KB}$ & $222 \mathrm{~KB}$ & $223 \mathrm{~KB}$ \\
House.png & $307 \mathrm{~KB}$ & $325 \mathrm{~KB}$ & $325 \mathrm{~KB}$ \\
Female.png & $287 \mathrm{~KB}$ & $309 \mathrm{~KB}$ & $310 \mathrm{~KB}$ \\
Female_2.png & $337 \mathrm{~KB}$ & $351 \mathrm{~KB}$ & $351 \mathrm{~KB}$ \\
baboon.png & $622 \mathrm{~KB}$ & $613 \mathrm{~KB}$ & $613 \mathrm{~KB}$ \\
Airplane.png & $415 \mathrm{~KB}$ & $418 \mathrm{~KB}$ & $418 \mathrm{~KB}$ \\
\hline
\end{tabular}

\section{CONCLUSION}

This paper is a combination of steganography and the mean filter algorithm. Most used images of image processing field used and compared with each other. Generally physical size of images stayed same after encryption. 30\% of images sizes differ from the original image. One of the image's size decreased after two different encryption process.

Steganography can be combined any other algorithms usefully. As a future work different filtering algorithms and also different image formats can be used and compared with each others.

\section{ACKNOWLEDGEMENT}

This work was supported by the Scientific and Technical Research Council of Turkey, under Project 118E682 and Research Fund of Istanbul University - Cerrahpasa under the project BYP-2019-33988.

\section{REFERENCES}

[1] Bukhari, Sadaf \& Shoaib Arif, Muhammad \& Anjum, M.R.\& Dilbar, Samia. (2016). Enhancing security of images by Steganography and Cryptography techniques. 531-534. 10.1109/INTECH.2016.7845050. 
[2] Kaur, Harpreet \& Kakkar, Ajay. (2017). Comparison of different image formats using LSB Steganography. 97-101. 10.1109/ISPCC.2017.8269657.

[3] Saritha, M \& M. Khadabadi, Vishwanath \& Sushravya, M. (2016). Image and text steganography with cryptography using MATLAB. 584-587.10.1109/SCOPES.2016.7955506.

[4] William K. Pratt, Digital Image Processing. Wiley, 1991.

[5] Anil K. Jain, Fundamentals of Digital Image Processing. Prentice Hall, 1989.

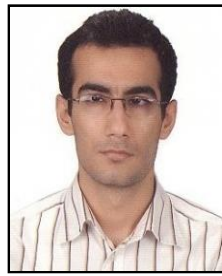

C. Tiken received his BCS degree in Computer Engineering from the Near East University, Cyprus in 2009. He received his Master's degree in Computer Engineering with specialization in deep learning from Istanbul University 2015, Turkey. Currently, he is pursuing $\mathrm{PhD}$ degree in image processing and cryptography from Istanbul University and he is a research assistant at Istanbul Arel University since 2014, Istanbul, Turkey. His research interests include image processing, cryptography and deep learning.

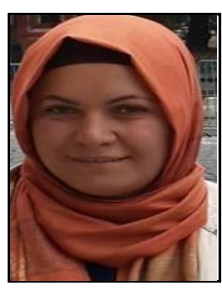

R. Samli received her $\mathrm{MSc}$ and $\mathrm{PhD}$ degrees in Computer Engineering from Istanbul University in 2006 and 2011 respectively. She received her master and $\mathrm{PhD}$ degrees about Artificial Neural Networks (ANN). She is an expert in ANN and also in software engineering. Currently she is working as an Assoc. Prof. Dr. in Istanbul University-Cerrahpasa. Her research interests include ANN, image processing and software engineering.

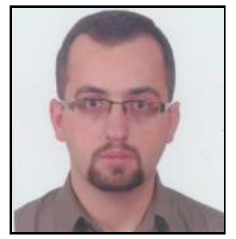

S. Sevgen received his $\mathrm{MSc}$ and $\mathrm{PhD}$ degrees in Computer Engineering from Istanbul University in 2003 and 2009 respectively. He received his degrees about image processing. Currently, he is working as an an Assoc. Prof. Dr. in Istanbul University-Cerrahpasa. His research interests include image processing, and Artificial Neural neteorks.
[6] Rafael C. Gonzalez and Richard E. Woods, Digital Image Processing. Addison-Wesley, 1992

[7] I. Pitas and A. N. Venetsanopoulos, Nonlinear Digital Filters: Principles and Applications. Kluwer Academic, 1990. https://doi.org/10.1007/978-1-4757-6017-0 